\title{
Análise da influência do posicionamento do fotopolimerizador sobre a rugosidade superficial da resina composta - estudo in vitro
}

\author{
Analysis of the influence of the photopolymerizer on the surface roughness of the \\ composite resin - in vitro study
}

\begin{abstract}
Mariana Menezes Vaz de Queiroz ${ }^{1}$, Andrea Nobrega Cavalcanti², Paula Mathias de Morais Canedo ${ }^{3 *}$
${ }^{1}$ Mestranda do Programa de Pós-Graduação em Processos Interativos dos Órgãos e Sistemas pelo Instituto de Ciências da Saúde da Universidade Federal da Bahia; ${ }^{2}$ Doutora em Clínica Odontológica pela Universidade Estadual de Campinas. Professora adjunta da Escola Bahiana de Medicina e Saúde Pública (BAHIANA). Professora Adjunta da Faculdade de Odontologia da Universidade Federal da Bahia (FOUFBA); ${ }^{3}$ Doutora em Clínica Odontológica pela Universidade Estadual de Campinas. Professora adjunta da Faculdade de Odontologia da Universidade Federal da Bahia (FOUFBA). Docente do Programa de Pós-Graduação Processos Interativos dos Órgãos e Sistemas do Instituto de Ciências da Saúde da Universidade Federal da Bahia.
\end{abstract}

\begin{abstract}
Resumo
Introdução: uma adequada polimerização da resina composta proporciona um bom desempenho clínico e propriedades físicas satisfatórias na restauração. A conversão de monômeros em polímeros depende não só da luz visível emitida pelo fotopolimerizador e do sistema fotoiniciador presente na resina composta, como também da quantidade de energia que alcança a resina composta e que está na dependência de como essa luz é aplicada sobre a resina. Objetivo: verificar a influência do posicionamento da ponta de um fotopolimerizador de amplo espectro sobre a rugosidade de duas resinas compostas. Metodologia: previamente à confecção dos corpos de prova, foi realizada a randomização de quatro grupos experimentais $(n=10)$ para locação dos corpos de prova, da resina Vittra APS (FGM) em duas apresentações (Vittra APS Esmalte-bleach e a Vittra APS Dentina A0) e duas variações do posicionamento da ponta do fotopolimerizador ( 0 e 20 ㅇ). Foram confeccionados quarenta corpos de prova cilíndricos em resina composta, na dimensão 6 × 1,5 mm. Valores da Ra foram registrados usando-se um rugosímetro e analisados estatisticamente (Shapiro-Wilk/ ANOVA). A rugosidade superficial ( $\mathrm{Ra}$ ) de topo de cada corpo de prova foi individualmente avaliada, sendo realizadas três leituras por superfície em posições diferentes. Resultados: não foi verificada significância estatística da interação entre os fatores, nem foram notadas diferenças estatisticamente significativas entre os fatores isolados. Dentre os posicionamentos analisados, constatou-se que o fotopolimerizador a 20 não alterou a rugosidade das resinas utilizadas. Conclusão: a variação da angulação da ponta do fotopolimerizador em $20^{\circ}$ não alterou a rugosidade superficial das resinas testadas.

Palavras-chave: Polimerização. Propriedades de Superfície. Saúde bucal. Restauração Dentária Permanente.
\end{abstract}

\begin{abstract}
Introduction: proper polymerization of the composite resin provides good clinical performance and satisfactory physical properties of the restoration. The conversion of monomers to polymers depends not only on the visible light emitted by the curing light and the photoinitiating system present in the composite resin, but also on the amount of energy that the composite resin reaches and is dependent on how that light is applied to the resin. Objective: to verify the influence of the tip positioning of a broad spectrum curing light on the roughness of two composite resins. Metodology: prior to the preparation of the specimens, four experimental groups ( $n=$ 10) were randomized to locate the specimens, Vittra APS resin (FGM) in two presentations (Vittra APS Enamel-bleach and Vittra APS Dentin $A O)$ and two variations of the curing tip positioning ( $\left(0\right.$ ) and $20^{\circ}$ ). Forty $6 \times 1.5 \mathrm{~mm}$ composite resin cylindrical specimens were made. Ra values were recorded using a roughness meter and statistically analyzed (Shapiro-Wilk/ANOVA). The top surface roughness $(R a)$ of each specimen was individually evaluated and three surface readings were taken at different positions. Results: no statistical significance of the interaction between the factors was verified, nor were statistically significant differences observed between the isolated factors. Among the positions analyzed, it was found that the curing light at 200 did not change the roughness of the resins used. Conclusion: variation of the curing angle of the curing light tip at $20^{\circ}$ did not change the surface roughness of the resins tested. Keywords: Polymerization. Surface properties. Oral health. Permanent dental restoration.
\end{abstract}

\section{INTRODUÇÃO}

Resinas compostas são materiais restauradores de amplo e progressivo uso na odontologia estética ${ }^{1}$. De-

Correspondente/Corresponding: *Paula Mathias de Morais Canedo Faculdade de Odontologia da Universidade Federal da Bahia. - End: Av. Araújo Pinho, no 72, Canela. CEP 40.110-912. Salvador, BA - Tel: (71) 99116-7275 — E-mail: pmathias@yahoo.com vido à capacidade de aliarem boa estética às excelentes propriedades mecânicas, são indicadas para dentes anteriores e posteriores ${ }^{2}$. As resinas compostas possuem, em sua composição, uma matriz orgânica e cargas inorgânicas quimicamente ligadas pelo agente de união, silano ${ }^{1}$.

As resinas compostas restauradoras, são, normalmente, materiais fotoativados que dependem de luz visível 
com características específicas de comprimento de onda e energia, bem como de um sistema fotoiniciador eficiente, para uma polimerização efetiva do material. O desempenho clínico e as propriedades físicas são fortemente influenciadas pelo grau de polimerização do material restaurador ${ }^{3,4}$. 0 processo da fotopolimerização se inicia a partir da fonte de luz, que ativa o sistema fotoiniciador presente na resina composta, o qual irá absorver a energia num espectro de luz visível, dando início à reação de polimerização ${ }^{3,5,6}$.

A luz emitida para fotoativar os materiais resinosos e realizar uma polimerização satisfatória, que consiste na máxima conversão de monômeros em polímeros, depende da quantidade de energia entregue pelo aparelho emissor de luz para o material resinoso. Essa energia é o produto da intensidade de potência do aparelho e a variação do tempo de aplicação dessa luz. O comprimento de onda - característica espectral da luz - é determinante para sensibilizar os agentes fotoiniciadores presentes na resina composta, já que cada fotoiniciador é sensibilizado por um ou alguns determinados comprimentos de onda ${ }^{3,7,8}$.

Além disso, existem fatores importantes que interferem na entrega da energia ao material restaurador pela luz, como a posição e a distância da ponta do fotopolimerizador. Essas duas variáveis afetam diretamente a quantidade de fótons que a resina composta irá receber, impactando no processo da polimerização ${ }^{7}$. Outros fatores clínicos que comprometem a dispersão de luz através da resina composta são as partículas de preenchimento e a espessura do incremento ${ }^{8,9}$.

Com a intenção de reduzir os aspectos negativos na fotopolimerização, foram desenvolvidas novas técnicas, além de sistemas de amplo espectro, com o uso de aparelhos com fotoativadores de diodos de luz (LED), capazes de ativar os fotoiniciadores presentes nos materiais resinosos ${ }^{5,8}$. As luzes de amplo espectro, a exemplo do fotopolimerizador Valo (Ultradent), melhoram a polimerização, afetando positivamente as propriedades das resinas compostas ${ }^{8}$. A fotoativação inadequada das resinas compostas pode estar relacionada às propriedades físicas, mecânicas, estéticas e biológicas inferiores, bem como à solubilidade no ambiente bucal, risco de microinfiltração aumentada, falhas na retenção e resposta pulpar adversa ${ }^{3,6,10}$.

A rugosidade da superfície em resina composta é uma ferramenta importante para indicar seu desempenho clínico e longevidade, e parece aumentada nos materiais que apresentam pobre conversão de monômeros em polímeros, pela perda superficial de parte de seus componentes ${ }^{11}$. Em função do exposto, o objetivo deste estudo é analisar a influência do posicionamento da ponta emissora de luz de um fotopolimerizador de amplo espectro sobre a rugosidade de duas resinas compostas. As hipóteses experimentais testadas são as de que, ${ }^{1}$ a alteração no posicionamento da ponta do fotopolimerizador aumenta a rugosidade superficial das resinas compostas testadas e que ${ }^{2}$ a resina composta de dentina apresenta maior rugosidade superficial que a resina composta indicada para o esmalte.

\section{METODOLOGIA}

\section{PREPARO DOS CORPOS DE PROVA}

Quarenta corpos de prova foram confeccionados com a resina Vittra APS, em duas apresentações: E-bleach, indicada para a camada de esmalte, e a DAO, indicada para a camada de dentina (Vittra APS E-bleach FGM, Joinville-SC e Vittra APS DA0, FGM, Joinville-SC). As resinas supracitadas foram investigadas em associação com duas variações do posicionamento ( 0 ㅇ e 20 ) da ponta do fotopolimerizador LED de amplo espectro (Valo Cordless $1500 \mathrm{~mW} / \mathrm{cm}^{2}$, Ultradent, South Jordan, EUA) (Figura 1).

Figura 1 - Organograma de distribuição dos grupos experimentais $(n=10)$.

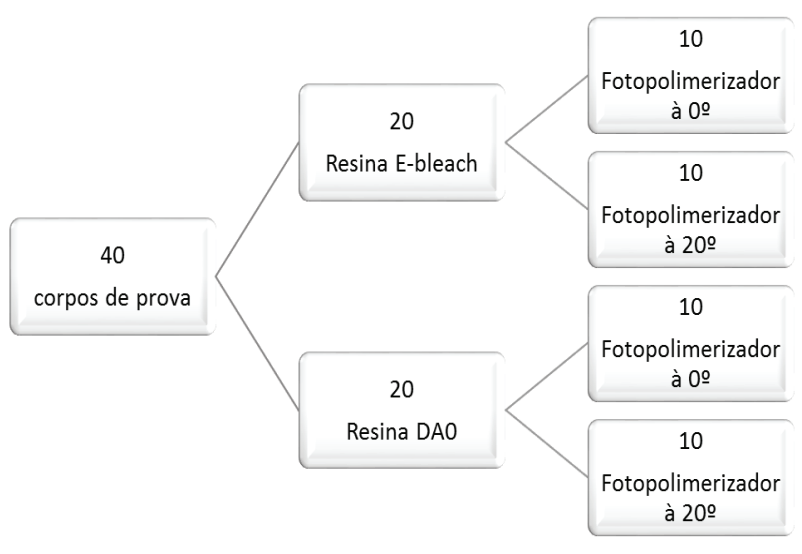

Fonte: Autoria própria.

Para a confecção de cada corpo de prova, a resina composta selecionada foi inserida em uma matriz de aço inox, com 1,5 mm de espessura e $6 \mathrm{~mm}$ de diâmetro. Sobre o conjunto, foi colocada uma tira matriz de poliéster e, sobre ela, um peso de $500 \mathrm{~g}$, que foi mantido por 30 segundos para permitir melhor acomodação do material. Após esse período, a superfície de topo foi fotoativada com luz LED (Valo Cordless $1400 \mathrm{~mW} / \mathrm{cm}^{2}$, Ultradent, South Jordan, EUA), respeitando-se as recomendações do fabricante, por 20 segundos para a resina E-bleach e 40 segundos para a resina DAO, obedecendo-se o posicionamento determinado da ponta do fotopolimerizador, através de um dispositivo confeccionado para garantir a inclinação padronizada e previamente escolhida para cada grupo experimental. 


\section{PROTOCOLOS DE EXPOSIÇÃO AO FOTOPOLIMERIZADOR}

Para a confecção dos corpos de prova, foi realizada a randomização de 4 grupos, estabelecidos com duas variações do posicionamento do fotopolimerizador. A exposição ocorreu obedecendo-se a um posicionamento predeterminado e padronizado da ponta emissora de luz do aparelho Valo.

Os protocolos utilizados no presente estudo estão descritos a seguir.

Protocolo controle - Os corpos de prova foram fotopolimerizados com a ponta do fotopolimerizador posicionada paralelamente ao material, ou seja, a 0 ㅇ do conjunto matriz + resina + tira de poliéster, em contato direto com a tira de poliéster. A resina E-bleach foi fotopolimerizada por 20 segundos, e a DA0 por 40 segundos, respeitando-se as recomendações do fabricante.

Protocolo 1 - Foi confeccionado um dispositivo em acrílico para estabelecer o posicionamento de 20 응 entre a ponta do fotopolimerizador e a resina inserida na matriz. Os corpos de prova foram fotoativados com a ponta do fotopolimerizador posicionado a 20 o do conjunto matriz + resina + tira de poliéster. A resina E-bleach foi fotopolimerizada por 20 segundos, e a DA0 por 40 segundos, respeitando-se, assim, as recomendações do fabricante.

Após a confecção dos corpos de prova, eles foram inseridos em cilindros de resina cristal, sendo, em seguida, armazenados a $37^{\circ} \mathrm{C}$ em umidade relativa, livres do contato com a luz (Figura 2).

Figura 2 - A: confeç̧ão dos corpos de prova a partir da inclusão da resina cristal. B: corpo de prova desenvolvido.

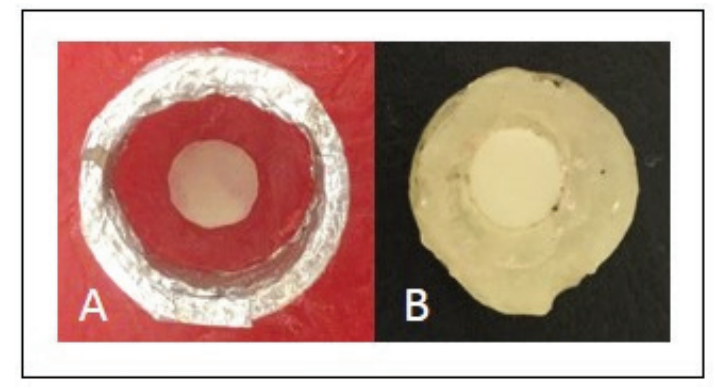

Fonte: Autoria própria.

Os corpos de prova tiveram a superfície de topo polida com o disco Polidor Diamantado para Resina CA Dura-Gloss One-Step (American Burrs, Palhoça - SC. Lote PI20170202), na dimensão $10 \times 2 \mathrm{~mm}$, sendo ele trocado a cada 13 corpos de prova.

Ao final de todos os processos, os corpos de prova foram colocados em umidade relativa de $37^{\circ} \mathrm{C}$. O Quadro 1 apresenta informações a respeito dos materiais utilizados.
Quadro 1 - Materiais utilizados, com identificação de seus respectivos fabricantes, lotes e sua composição química básica

\begin{tabular}{|l|l|}
\hline Material (fabricante); lote & Composição química básica \\
\hline $\begin{array}{l}\text { Resina composta (Vittra APS } \\
\text { E-bleach, FGM, } \\
\text { Joinville - SC); Lote 271118. }\end{array}$ & $\begin{array}{l}\text { Ingredientes ativos: mistura monomé- } \\
\text { rica contendo monômeros tipo UDMA } \\
\text { (trietileno glicol dimetacrilato), com- } \\
\text { posição fotoiniciadora APS (Advanced } \\
\text { Polymerization System), coiniciadores, } \\
\text { estabilizante e silano. } \\
\text { Ingredientes inativos: carga de zircô- } \\
\text { nia, sílica e pigmentos. }\end{array}$ \\
\hline Resina composta (Vittra APS & $\begin{array}{l}\text { Ingredientes ativos: mistura monomé- } \\
\text { rica contendo monômeros tipo UDMA } \\
\text { DA0, FGM, } \\
\text { Joinville - SC); Lote 260618. }\end{array}$ \\
$\begin{array}{l}\text { (trietileno glicol dimetacrilato), com- } \\
\text { posição fotoiniciadora APS (Advanced }\end{array}$ \\
$\begin{array}{l}\text { Polymerization System), coiniciadores, } \\
\text { estabilizante e silano. } \\
\text { Ingredientes inativos: carga de zircônia, } \\
\text { sílica e pigmentos. }\end{array}$ \\
\hline
\end{tabular}

Fonte: Autoria própria.

\section{AVALIAÇÃO DA RUGOSIDADE SUPERFICIAL}

A rugosidade da superfície de topo de cada corpo de prova foi individualmente avaliada com auxílio do rugosímetro (Surftest 3000, Mitutoyo Sul Americana, São Paulo, SP, Brasil). O valor considerado para cada leitura foi a média aritmética entre picos e vales (Ra), registrada num trecho de $1,25 \mathrm{~mm}$, numa velocidade de $0,1 \mathrm{~mm} / \mathrm{s}$. Foram realizadas três leituras por superfície, em posições diferentes, para maior abrangência da área testada. 0 valor de rugosidade superficial foi obtido pela média aritmética das três leituras.

\section{ANÁLISE ESTATÍSTICA}

Para cálculo da amostra, utilizou-se como critério, em primeiro lugar, a realização da Análise de Variância para comparação de média de 4 grupos independentes, assumindo um poder de teste de $80 \%$ com nível de significância de $5 \%$ e tamanho de efeito $f=0,5^{15}$.

Inicialmente, foi realizada a análise exploratória dos dados de rugosidade para verificação da presença de normalidade (Shapiro-Wilk; $p>0,05$ ) e demais parâmetros da ANOVA. A análise estatística inferencial foi realizada pela ANOVA de 2-critérios, da resina $x$ fotopolimerizador. As análises foram realizadas no programa estatístico SAS, versão 9.1, com nível de significância de 5\%.

\section{RESULTADOS}

A Tabela 1 apresenta a média e o desvio-padrão obtidos pela variável rugosidade. 
Tabela 1 - Média (desvio-padrão) dos valores de rugosidade.

\begin{tabular}{lccc}
\hline Resina & Fotopolimerizador & Média (desvio-padrão) & \\
\hline E-bleach & $0^{\circ}$ & $1.17(0.30)$ & $\mathrm{A}$ \\
& $20^{\circ}$ & $1.20(0.41)$ & $\mathrm{A}$ \\
$\mathrm{DAO}$ & $0^{\circ}$ & $1.08(0.24)$ & $\mathrm{A}$ \\
& $20^{\circ}$ & $1.08(0.33)$ & $\mathrm{A}$ \\
\hline
\end{tabular}

Letras distintas representam significância estatística entre os grupos (1-way ANOVA/Tukey, alfa $=5 \%$ ).

Fonte: Autoria própria.

De acordo com a análise, não foi verificada significância estatística da interação entre resina $x$ fotopolimerizador $(p=0,87)$; nem foram notadas diferenças estatisticamente significativas entre os fatores isolados (resina: $p=0,31$; fotopolimerizador: $p=0,91$ ).

\section{DISCUSSÃO}

A fotoativação inadequada das resinas compostas pode comprometer suas propriedades e, consequentemente, seu desempenho clínico e sua durabilidade. As superfícies rugosas e ásperas favorecem o acúmulo de placa bacteriana e a modificação na coloração do material restaurador ${ }^{12-14}$. Segundo Bollen, Lambrechts e Quirynen ${ }^{11}$, a rugosidade superficial, com valor de $0,2 \mu \mathrm{m}$, é o limite máximo aceitável para se evitar retenção de biofilme, pois valor superior a esse aumenta o risco de desenvolvimento de lesões cariosas adjacentes à restauração e doenças periodontais. Vale ressaltar que Jones, Billington e Pearson ${ }^{16}$ relatam que, clinicamente, os pacientes podem detectar a rugosidade superficial ( $R a$ ) quando os valores são maiores que $0,3 \mu \mathrm{m}$.

$\mathrm{Na}$ presente investigação, os resultados mostraram que o tipo de resina - esmalte ou dentina - e a angulação da ponta do fotoativador, em $20^{\circ}$ ou $0^{\circ}$ foram variáveis que não interferiram na rugosidade superficial das resinas compostas testadas (Vittra APS E-bleach e Vittra APS DA0). Assim, as hipóteses experimentais do presente estudo foram todas negadas, já que não se observaram diferenças significativas entre nenhum dos grupos experimentais.

A resina composta tem, em sua composição, basicamente, cargas inorgânicas e uma matriz orgânica quimicamente ligadas pelo agente de união silano. As resinas compostas que apresentam partículas de carga menores evidenciam aspereza superficial menor do que as que possuem partículas de carga maiores. A resina Vittra APS, utilizada nesta investigação, é um compósito nanoparticulado, de alta estética, radiopaco, e sua carga é composta por nanoesferas de um complexo de zircônia, com tamanho de cargas manométricas de $100-200 \mathrm{~nm}$, sendo o conteúdo total de carga inorgânica, em peso, de $72 \%$ a $82 \%$ ( $52 \%$ a $60 \%$ em volume $)^{17}$. O conteúdo, o formato e o tipo de carga conferem a esse compósito desempenho mecânico, resistência ao desgaste e estética, o que é traduzido em elevado brilho e polimento ${ }^{17}$.

As resinas fotopolimerizáveis são compostas de sistemas fotoiniciadores que absorvem a luz para desenvolver estágios excitados que iniciam a polimerização ${ }^{18}$. Para isso, é necessário o uso de uma fonte de luz que apresente um espectro visível, compatível com as características de absorção dos fotoiniciadores, para que a absorção ocorra e se inicie a reação de polimerização ${ }^{3,5,6}$. Como polimerização ideal do compósito, entende-se uma elevada conversão de seus monômeros em cadeias poliméricas entrelaçadas, que formam os polímeros ${ }^{19}$.

A quantidade de energia entregue ao compósito pela ponta do aparelho fotoativador influencia significativamente a polimerização, e é definida pelo produto da intensidade de potência $\left(1.200 \mathrm{mw} / \mathrm{cm}^{2}\right.$, valor representativo para os fotopolimerizadores contemporâneos) e do tempo (segundos) de aplicação da luz ${ }^{3,78}$. Outro aspecto a ser levado em consideração é o comprimento de onda da luz, que pode variar entre 400 e $500 \mathrm{~nm}$, e está diretamente relacionado à capacidade de sensibilização dos fotoiniciadores pela luz, independentemente do valor da energia da luz depositada sobre o material restaurador resinoso ${ }^{3,8}$.

Ressalta-se que existem variações nos sistemas de fotopolimerização das resinas e, com isso, variação na sensibilidade dos fotoiniciadores a um determinado comprimento de onda da luz para sua ativação ${ }^{8}$. Como exemplo, a canforoquinona é mais sensível à luz com $468 \mathrm{~nm}$ de comprimento de onda ou abaixo de $320 \mathrm{~nm}$; já os fotoiniciadores alternativos (Lucirin TPO e Ivocerin) são mais reativos a comprimentos de onda de luz mais baixos, perto de $410 \mathrm{~nm}^{8}$. De acordo com o Perfil Técnico Vittra APS ${ }^{17}$, o desenvolvimento do novo sistema de fotopolimerização incorporado a essa resina, o sistema fotoiniciador APS (Advanced Polymerization System), permite maior profundidade de cura e grau de conversão, resultando, portanto, em uma matriz polimérica de alta resistência. O sistema APS - consistente na combinação de diferentes fotoiniciadores, dentre eles uma pequena quantidade de canforoquinona - possui o espectro de absorção para sua ativação no comprimento de onda de 400-500 $\mathrm{nm}^{20}$.

Em conformidade com a literatura, uma posição adequada da ponta do fotopolimerizador é necessária para que a resina receba uma quantidade de fótons suficientes, para, através da energia de luz recebida, ela possa ser fotopolimerizada corretamente ${ }^{7}$. Salienta-se que as partículas de preenchimento e a espessura do incremento comprometem a dispersão de luz através da resina composta ${ }^{8,9}$, pois partículas maiores promovem maior dispersão de luz, consequentemente, menor quantidade de luz será transmitida através da resina ${ }^{8}$.

Pesquisas relatam que os aparelhos com fotoativadores de diodos de luz (LED) são capazes de ativar adequadamente os fotoiniciadores dos sistemas resinosos utilizados ${ }^{5,8}$. O dispositivo de LED tem uma faixa espectral estreita, com um pico de cerca de $470 \mathrm{~nm}$, que coincide com o comprimento de onda de absorção ideal para a ativação do fotoiniciador canforoquinona, resultando em aumento do grau de conversão do compósito ${ }^{21}$. Segundo 
Shimokawa ${ }^{8}$, as luzes de amplo espectro, a exemplo do fotopolimerizador Valo (Ultradent), são capazes de melhorar positivamente as propriedades das resinas compostas, devido à sua eficácia na transmissão da luz, ocasionando uma polimerização eficiente. De acordo com o fabricante, o fotopolimerizador Valo utiliza um diodo emissor de luz (LED) de multicomprimento de onda (395-400nm), para produzir uma luz de alta intensidade, capaz de polimerizar todos os materiais dentários fotopolimerizáveis. Aguiar et al. ${ }^{21}$ evidenciaram que o dispositivo LED obteve maior grau de conversão do compósito, quando comparado a outro dispositivo.

Dessa maneira, o fato de o posicionamento do fotopolimerizador a 20으, no presente estudo, não evidenciar aumento no valor da rugosidade superficial das resinas compostas de esmalte e dentina, quando comparado ao posicionamento a 0 o da ponta do fotopolimerizador, pode ser justificado pelo potencial e pela eficácia do fotopolimerizador de amplo espectro Valo em transmitir a luz através das resinas, portanto, converter adequadamente monômeros em polímeros, independentemente do conteúdo orgânico da resina e das partículas de carga 22, 23. Ainda segundo Gonulol, Ozer e Tunc ${ }^{23}$, o Valo, em alta potência $\left(1400 \mathrm{mw} / \mathrm{cm}^{2}\right)$, utilizado para polimerizar uma resina composta nanoparticulada, mostrou eficiência na polimerização adequada do material restaurador. Vale ressaltar que a resina Vittra APS é composta por partículas de nanoesfera de zircônia, as quais permitem que menos luz incidida sobre ela seja dispersada e que mais luz seja transmitida através dela, promovendo, assim, uma polimerização eficiente e consistente do compósito, com bons resultados de suas propriedades físicas.

Estudos que avaliem outros dados, além da rugosidade, e que também possam explorar outras variáveis importantes, como maiores inclinações, diferentes distâncias e posicionamentos da ponta do aparelho devem ser conduzidos, para que possam determinar a real influência da posição do fotopolimerizador sobre a polimerização de diferentes resinas compostas.

\section{CONCLUSÃO}

No presente estudo, verificou-se que a rugosidade superficial das resinas compostas de esmalte e dentina avaliadas não foi afetada pela alteração das angulações de $0^{\circ}$ e $20^{\circ}$ da ponta do fotopolimerizador Valo. Além disso, não foi observada diferença estatisticamente significativa entre os dois tipos de resina composta testada - esmalte e dentina.

\section{REFERÊNCIAS}

1. DE QUEIROZ, M. M. V. et al. Effect of erosion and methods for its control on the surface roughness of composite resin. Rev. Odonto. Ciênc., Porto Alegre, v.32, n.2, p.88-93, 2017.

2. MUNCHOW, E. A. et al. Effect of acidic solutions on the surface degradation of a micro-hybrid composite resin. Braz. Dent. J., Ribeirão Preto, v. 25, n. 4, p. 321-326, 2014.
3. KNEZEVIC, A. et al. Degree of conversion and temperature rise during polymerization of composite resin samples with blue diodes. J. Oral Rehabil., Oxford, v. 28, p.586-591, 2001.

4. SOARES, C. C. P. et al. Eficácia da polimerização de uma resina composta fotopolimerizada por aparelhos de luz halógena e LED da clínica integrada do curso de odontologia da UFES. UFES Rev. Odontol., Vitória, v.7, n.3, p. 58-65, 2005.

5. CCAHUANA-VÁSQUEZ, R. A. et al. Influência do tipo de ponteira condutora de luz de aparelhos LED na microdureza das resinas compostas. Rev. Odonto. UNESP, São Paulo, v.33, n. 2, p. 69-73, 2004.

6. SANTOS, M. J. M. C. et al. Novos conceitos relacionados à fotopolimerização das resinas compostas. JBD, J. Bras. Dent. Estét., [s.I.], v.1, n. 1, p.14-21, 2002.

7. KARACOLAK, G. et al. Influenceof incremente thicknesson radiant energy and micro hardness of bulkfill resin composites. Dent. Mater. J., Tokyo, v. 37, n.2, p. 206-213, 2018.

8. SHIMOKAWA, C. A. K. Influence of emission spectrum and irradianceon light curing of resin-based composites. Oper. Dent., Seattle, v. 42, n. 5, p. 537-547, 2017.

9. TARLE, Z. et al. Influence of irradiation time on subsurface degree of conversion and microhardness of high-viscosity bulk-fill resin composites. Clin. Oral Investig., Berlin, v.19, n. 4, p. 831-840, 2014.

10. SEGRETO, D. R. et al. Influence of photoinitiator and light-curing source on bond strength of experimental resin cements to dentin. Braz. Dent. J., Ribeirão Preto, v. 27, n. 1, p. 83-89, 2016.

11. BOLLEN, C. M. L.; LAMBRECHTS, P.; QUIRYNEN, M. Comparison of surface roughness of oral hard materials to the threshold surface roughness for bacterial plaque retention: a review of the literature. Dent. Mater., Washington, v. 13, n. 4, p. 258-269, 1997.

12. SOARES, L. E. S.; DE CARVALHO FILHO, A. C. B. Protective effect of fluoride varnish and fluoride gel on enamel erosion: roughness, SEM-EDS, and u-EDXRF. Microsc. Res. Tech., New York, v. 78, n. 3, p. 240-248, 2015.

13. ELKASSAS, D.; ARAFA, A. Remineralising efficacy of different calcium-phosphate and fluoride based delivery vehicles on artificial caries like enamel lesions. J. Dent., [s.I], v. 42, n. 4, p. 466-474, 2014.

14. WILDER JUNIOR, A. D. et al. Effect of finishing technique on the microleakage and surface texture of resin-modified glass ionomer restorative materials. J. Dent., [s.I], v. 28, n. 5, p. 367-373, 2000.

15. COHEN, J. Statistical power analysis for the behavioral sciences. 2 ed. Hillsdale: Lawrence Erlbaum Associates, 1988.

16. JONES, C. S.; BILLINGTON, R. W.; PEARSON, G. J. The in vivo perception of roughness of restorations. Br. Dent. J., London, v.196, p. 42-45, 2004.

17. PERFIL TÉCNICO DA VITTRA APS. Disponível em: http://www.fgm. ind.br/site/wp-content/uploads/2017/03/Perfil-Tecnico-Vittra.pdf. Acesso em: 23 ago. 2019.

18. HADIS, M. A.; SHORTALL, A. C.; PALIN, W. M. Competitive light absorbers in photoactive dental resin-based materials. Dent. Mater., Washington, v.28, n. 8, p. 831-841, 2012. DOI:10.1016/j.dental.2012.04.029.

19. RIBEIRO, B. C. I. et al. Degree of conversion of nanofilled and microhybrid composite resins photo-activated by diferente generations of LEDs. J. Appl. Oral Sci., Bauru, v. 20, n. 2, p. 212-217, 2012.

20. REPENSE seu conceito de fotopolimerização. Disponível em: https:// cdn.dentalspeed.com/manual/Materia\%20-\%20APS.pdf. Acesso em: 23.ago. de 2019. 
21. AGUIAR, F. H. et al. Effect of different light-curing modes on degree of conversion, staining susceptibility and stain's retention using different beverages in a nanofilled composite resin. J. Esthet Restor. Dent., Hamilton, v. 23, n. 2, p. 106-114, Apr. 2011. DOI: 10.1111/j.17088240.2011.00406.x.

22. SANTINI, A. et al. Degree of conversion and microhardness of TPO- -containing resin-based composites cured by polywave and monowave LED units. J. Dent., [s.I], v. 40, n.7, p.577-584, July 2012.

23. GONULOL, N.; OZER, S.; TUNC, E. S. Effect of a third- generation LED LCU on microhardness of tooth-colored restorative materials. Int. J. Paediatr. Dent., Oxford, v. 26, n. 5, p.376-382, 2015. DOI:10.1111/ipd.12213.

Submetido em: 04/11/2019

Aceito em: 30/11/2019 